\title{
A Miniaturized Patch Antenna by Using a CSRR Loading Plane
}

\author{
Mehrab Ramzan ${ }^{1}$ and Kagan Topalli ${ }^{2}$ \\ ${ }^{1}$ Department of Electrical and Electronics Engineering, Bilkent University, 06800 Ankara, Turkey \\ ${ }^{2}$ National Nanotechnology Research Center (UNAM), Bilkent University, 06800 Ankara, Turkey \\ Correspondence should be addressed to Kagan Topalli; topalli@unam.bilkent.edu.tr
}

Received 15 June 2015; Revised 16 July 2015; Accepted 26 July 2015

Academic Editor: Maria E. De Cos

Copyright (C) 2015 M. Ramzan and K. Topalli. This is an open access article distributed under the Creative Commons Attribution License, which permits unrestricted use, distribution, and reproduction in any medium, provided the original work is properly cited.

\begin{abstract}
This paper presents a design methodology for the implementation of a miniaturized square patch antenna and its circuit model for $5.15 \mathrm{GHz}$ ISM band. The miniaturization is achieved by employing concentric complementary split ring resonator (CSRR) structures in between the patch and ground plane. The results are compared with the traditional square patch antenna in terms of area, bandwidth, and efficiency. The area is reduced with a ratio of $1 / 4$ with respect to the traditional patch. The miniaturized square patch antenna has an efficiency, bandwidth, and reflection coefficient of $78 \%, 0.4 \%$, and $-16 \mathrm{~dB}$, respectively. The measurement and circuit modeling results show a good agreement with the full-wave electromagnetic simulations.
\end{abstract}

\section{Introduction}

Microstrip patch antennas are preferred due to their low cost, light weight, and ease of integration in many microwave applications. However, there is demand on the reduction of antenna size in particular applications such as some military and commercial wireless communication systems where the system size and profile are a constraint. Over the past few years, significant amount of work has been done to reduce the size of the patch antennas. One of the traditional methods is to use materials with high permittivity [1] which yields a size reduction up to $30-50 \%$. However high permittivity substrates lead to high cost and suffer from surface waves which degrade the radiation characteristics of the antenna by increasing significant amount of side lobes [2-4]. To suppress the surface waves a number of techniques are proposed in [57]. Among them, the most famous remedy is to surround the antenna structure with band gap structures to prevent energy from being trapped inside the substrate. However, forming a band gap by making periodically placed holes occupies a large area making this approach impractical for miniaturized antennas. Another technique to reduce the patch size is to employ RIS (reactive impedance substrate) as discussed in [8] where Trans-Tech MCT-25 magnesium calcium titanate composition $\left(\varepsilon_{r}=25\right)$ is used as a RIS substrate. However using such a high permittivity and nonstandard substrate increases the cost significantly.

In some other research efforts, it is proposed to achieve miniaturization by increasing the path of the current on the patch antenna. It is reported in [9] that a reduction up to $36 \%$ is achieved by using slits. Some of the most common methods which can be employed to reduce the size of the antenna are inserting slots [9], shorting pin [10], corrugation structure [11], and iris structures [12]. However, all of these methods have some limitations such as low performance and manufacturing complexity.

In order to reduce the size of patch antennas complementary split ring resonators (CSRRs) on ground plane [1315] are proposed typically providing average reduction of about $30 \%$ in area which is accompanied with a decrease of front to back ratio up to $1 \mathrm{~dB}$. They also increase the crosspolarization to the same level as the copolarization. In this paper, miniaturized square patch antenna is proposed for ISM $5.15 \mathrm{GHz}$ band by using a simple design methodology. The size reduction is achieved by employing a CSRR based metamaterial plane in between the patch and ground plane without degrading the front to back ratio significantly. The measurement results indicate that the area is reduced with a ratio of $1 / 4$ compared to a traditional patch at the expense of an acceptable degradation in efficiency and bandwidth. 


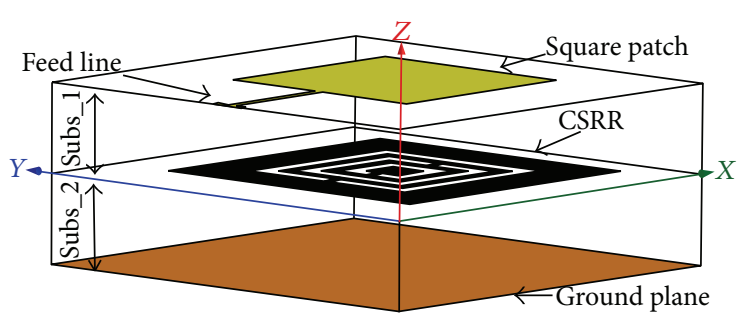

FIGURE 1: 3D side view of miniaturized antenna consisting of CSRR (metamaterial) layer between the square patch and ground plane.

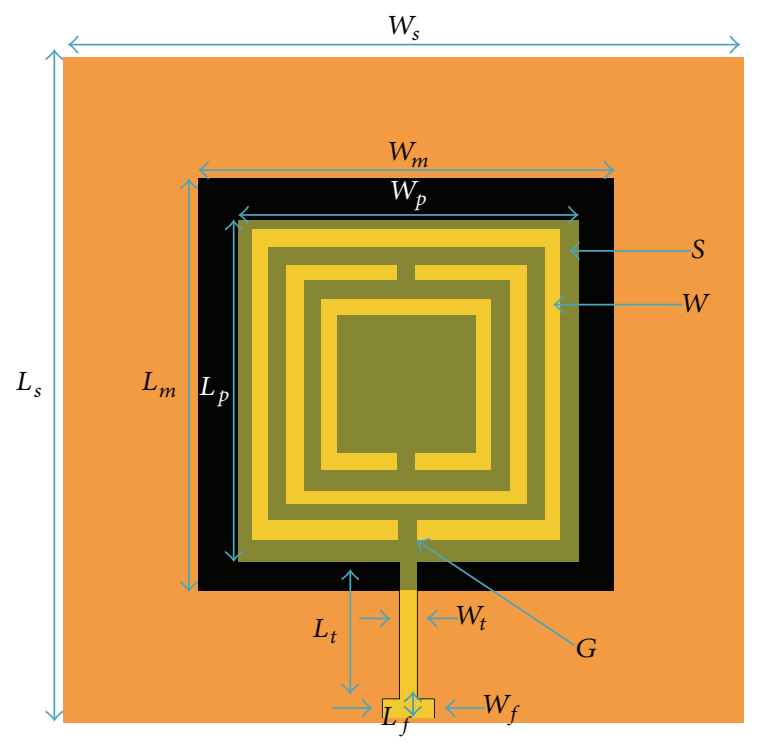

FIGURE 2: Top view of miniaturized antenna with physical parameters.

To the authors' knowledge the approach presented in this work, which is also supported with measurements and circuit model, is applied on square patches for the first time. The following sections present the antenna design approach and simulation results in comparison with the measurement results and finally a simple transmission line model of miniaturized patch is discussed.

\section{Antenna Design}

The miniaturized patch antenna consists of two separate substrates where the metamaterial plane is sandwiched in between, as shown in Figure 1. The top and bottom planes on the surfaces of this stack of substrates form the patch antenna and the ground plane, respectively. Rogers RT/Duroid 6002 having relative permittivity of $\varepsilon_{r}=2.92$, loss tangent of $\tan \delta=0.002$, and height of $h=0.762 \mathrm{~mm}$ is used for the design. The top view of the miniaturized antenna geometry is shown in Figure 2. The metamaterial plane is composed of a metal plane with CSRR pattern on it. Three concentric CSRRs are fit considering the fabrication tolerances. Two RT/Duroid 6002 with $0.762 \mathrm{~mm}$ heights are used and denoted as subs_l and subs_2 in Figure 2. Considering the analysis and
TABLE 1: Physical parameters of the traditional antenna with quarterwave transformer and miniaturized patch antennas.

\begin{tabular}{lcc}
\hline Parameters & $\begin{array}{c}\text { Traditional patch } \\
(\mathrm{mm})\end{array}$ & Miniaturized patch $(\mathrm{mm})$ \\
\hline$L_{s}$ & 40 & 28 \\
$W_{s}$ & 40 & 28 \\
$L_{p}$ & 15.95 & 8 \\
$W_{p}$ & 15.95 & 8 \\
$L_{t}$ & 9 & 14.4 \\
$W_{t}$ & 0.7 & 1.2 \\
$L_{f}$ & 4 & 1 \\
$W_{f}$ & 4 & 4 \\
$L_{m}$ & - & 14.4 \\
$W_{m}$ & - & 14.4 \\
$S$ & - & 0.51 \\
$G$ & - & 0.3 \\
$W$ & - & 0.25 \\
\hline
\end{tabular}

the proposed models of SRR structure discussed in $[14,16-$ 18 ], the gap $G$ of the SRR acts as a capacitor and length of the SRR increases the inductive effects. CSRR is the dual structure of SRR. The metamaterial plane in between two substrates acts as the loading element decreasing the electrical size of the antenna. In order to assess the level of miniaturization, a square shaped traditional patch is also implemented without the metamaterial plane.

\section{Results and Discussion}

The optimization of antennas is carried out by using parametric analyses of Ansys HFSS. A traditional patch, where the metamaterial plane is not included in between two substrates, is chosen as the reference antenna. Table 1 gives the physical parameters of the antennas. The miniaturized antennas are implemented by attaching two separate substrates: The first one, subs_l, has the patch pattern and the second one, subs_2, has the CSRR (metamaterial layer) pattern on the top surface and the ground plane at the bottom. LPKF rapid PCB prototyping tool [19] is used to pattern the layers with a precision of about $0.2 \mathrm{~mm}$. The substrates are aligned by using thru holes and separate substrates are fixed using Kapton tapes as shown in Figure 3. Figure 4 shows the measured and simulated reflection coefficients of the traditional patch and miniaturized patch which has an area of $1 / 4$ of the traditional 


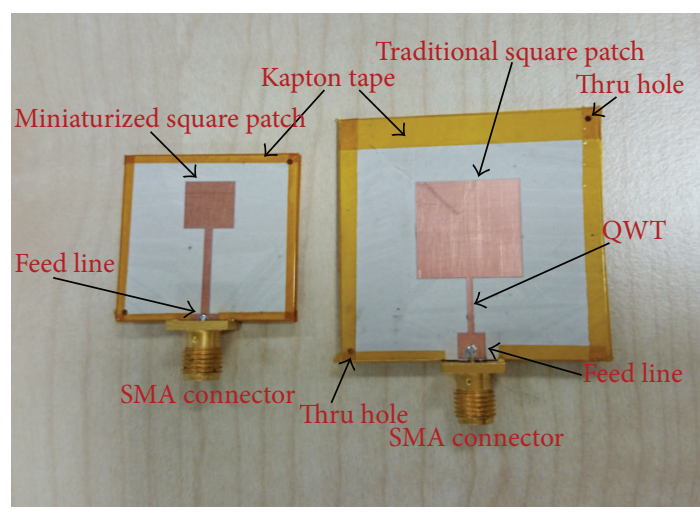

FIGURE 3: A photograph of the fabricated designs of traditional patch with quarter-wave transformer and miniaturized patch with longer feed line.

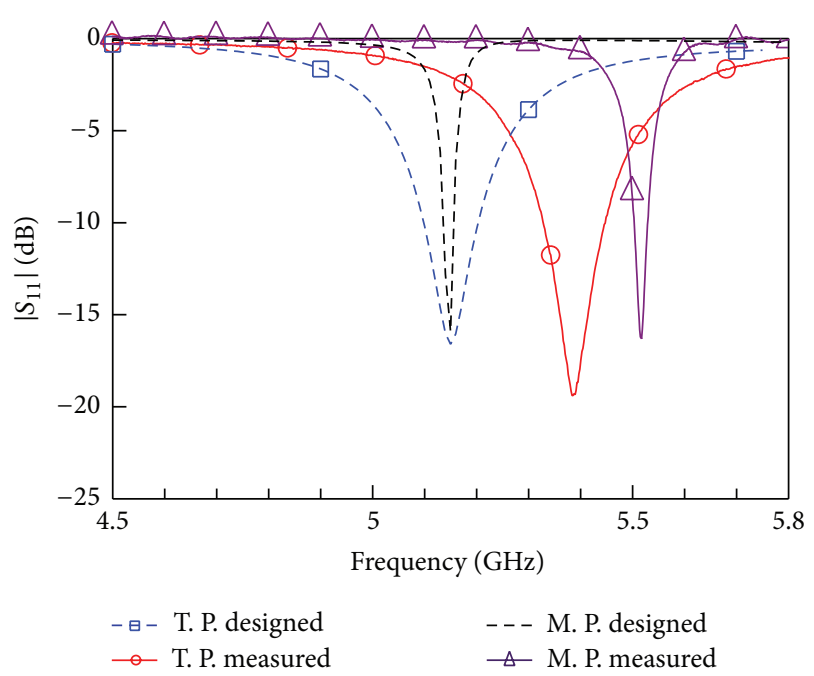

FIGURE 4: Measured and simulated reflection coefficient comparison of traditional antenna with quarter-wave transformer and miniaturized antenna with longer feed line.

patch. It is evident from the results that the miniaturization has led to the reduction of the $-10 \mathrm{~dB}$ bandwidth of the antenna from $2 \%$ to $0.4 \%$. The measurement results also indicate that there is a shift between the measured and simulated results of both antennas. The main reason behind this shift is the unwanted air gap remaining in between the substrates during the prototyping process. In order to verify the effect of the air gap on the resonant frequency, the simulation models are modified by including the air gap in between subs_l and subs_2. Figure 5 compares the results of measured and modified simulations for the reflection coefficients. A perfect agreement is achieved by employing an air gap of $0.11 \mathrm{~mm}$ and $0.045 \mathrm{~mm}$ in between subs_l and subs_2 of traditional antenna and miniaturized patch, respectively. It should be noted here that miniaturized antenna is quite sensitive to air gap since the characteristics of the CSRR region are strongly affected by the presence of air. Since only miniaturized patch incorporates CSRR layer, which increases

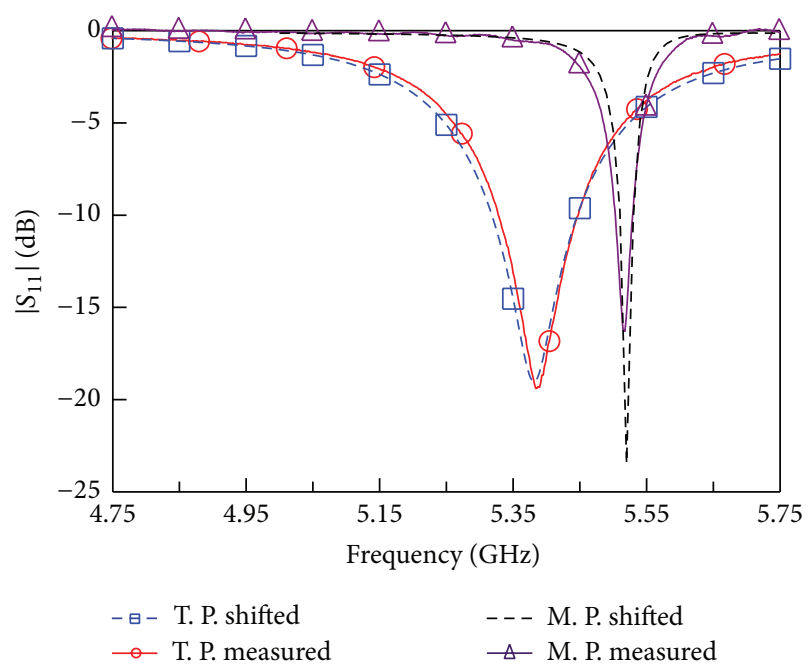

FIGURE 5: Measured and shifted simulated reflection coefficient comparison of the traditional antenna with quarter-wave transformer and miniaturized antenna with longer feed line.

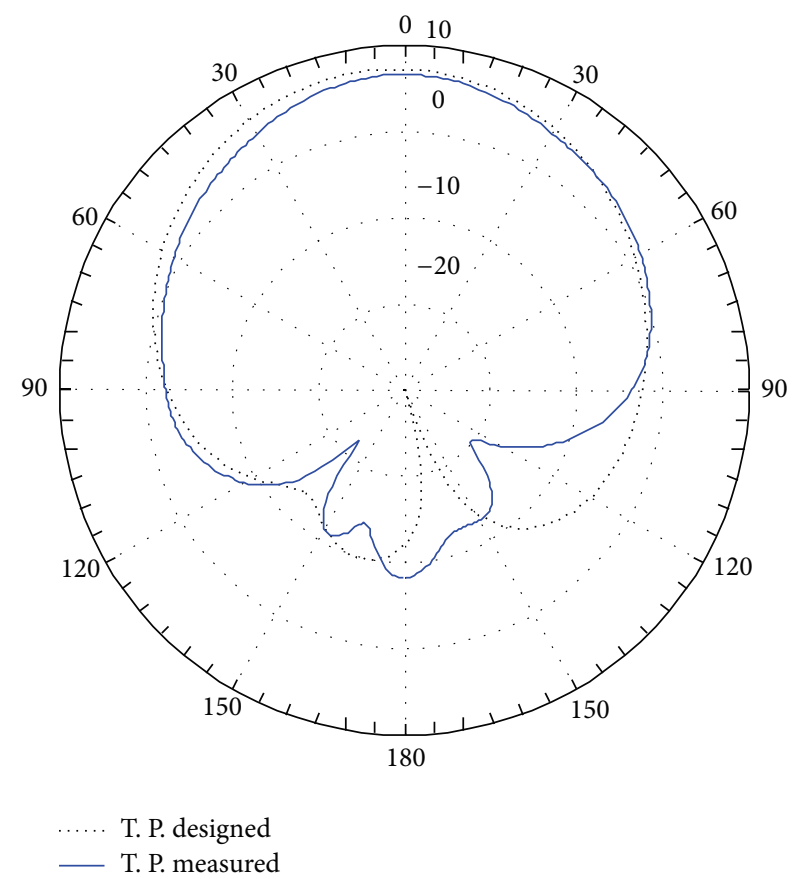

FIGURE 6: Simulation and measurement results for $E$-plane gain of traditional patch with quarter-wave transformer (frequency: $5.38 \mathrm{GHz})$.

its sensitivity to the air gap, that is why the measured reflection coefficient of miniaturized patch has moved to higher frequency as compared to the traditional patch antenna as shown in Figure 5. Figures 6 and 7 show the simulated and measured gain patterns of traditional patch in $E$ - and $H$ planes, respectively. The measurements are carried out using Satimo StarLab $18 \mathrm{GHz}$ antenna measurement system. The simulated and measured results agree well verifying that the manufacturing method of stacking multiple substrates can 


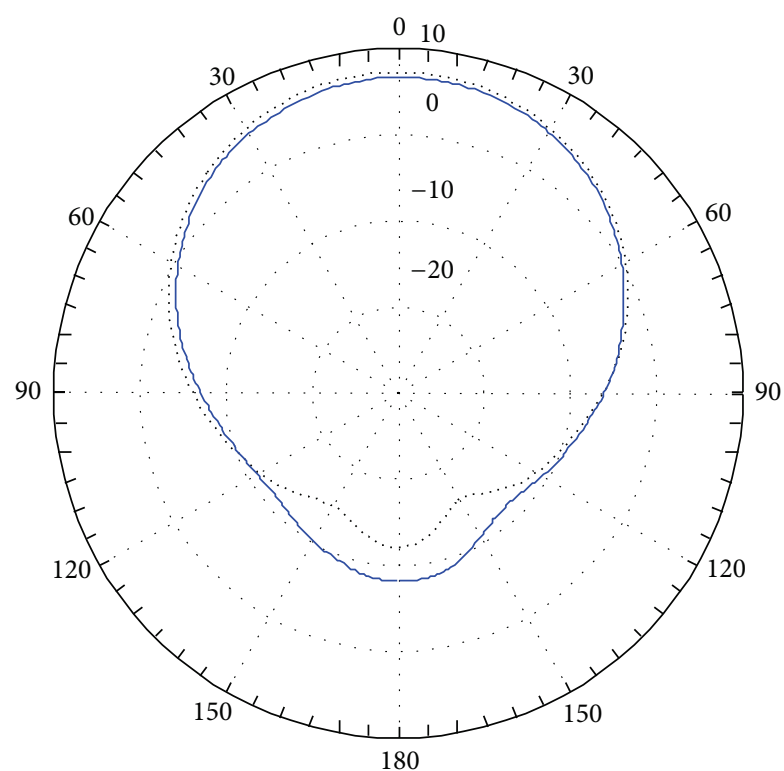

.... T. P. designed

- T. P. measured

FIgURE 7: Simulation and measurement results for $H$-plane gain of traditional patch with quarter-wave transformer (frequency: $5.38 \mathrm{GHz})$.

work effectively for the traditional patch. Figures 8 and 9 present the simulated and measured gain patterns in $E$ - and $H$-plane of miniaturized patch. There is a slight decrease observed in the gain of miniaturized patch which is due to a slight displacement of metamaterial layer along the feed line during the fabrication. Figure 10 shows the change of the antenna gain as the metamaterial layer is shifted towards the feed. It is noted that displacement of even less than $1 \mathrm{~mm}$ from the patch center towards the feed line reduces the gain tremendously. As the CSRR layer position determines the current distribution on the miniaturized patch, the radiation patterns being highly dependent on surface current distribution will also change. The detailed discussion on the change of current distribution with respect to CSRR layer is given in the following sections of paper. Figures 11 and 12 show how the gain patterns are distorted if CSRR layer is not placed accurately beneath the miniaturized patch. The manufacturing accuracy can be improved by using photolithographic and wafer bonding processes, where double sided patterning and bonding is achievable on lowloss substrate with alignment accuracy in the order of a few micrometers. After miniaturization the front to back ratio is reduced from $23 \mathrm{~dB}$ to $21.5 \mathrm{~dB}$. The co- to cross-polarization ratio of traditional patch in $E$ - and $H$-plane are 47 and $30 \mathrm{~dB}$, respectively. For miniaturization of patch antenna the co- to cross-polarization ratio in $E$ - and $H$-plane are reduced to 43 and $27 \mathrm{~dB}$, respectively. With this level of miniaturization the miniaturized values of front to back ratio and co- to crosspolarization ratio are practically acceptable and comparable to the results given in $[7,20,21]$.

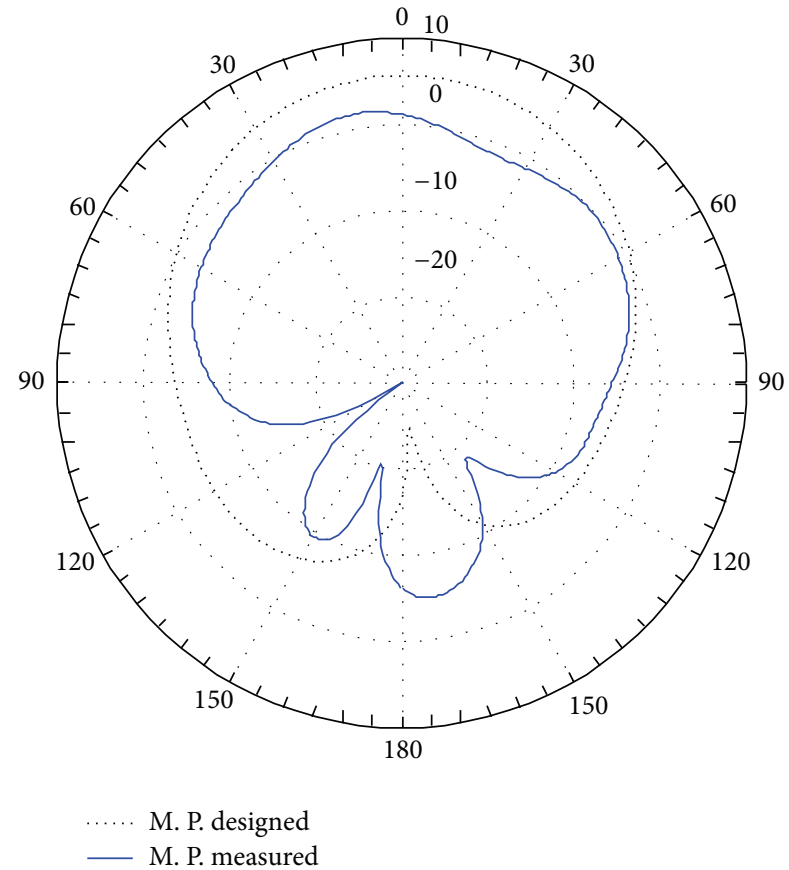

FIgURE 8: Simulation and measurement results for $E$-plane gain of miniaturized patch with longer feed line (frequency: $5.51 \mathrm{GHz}$ ).

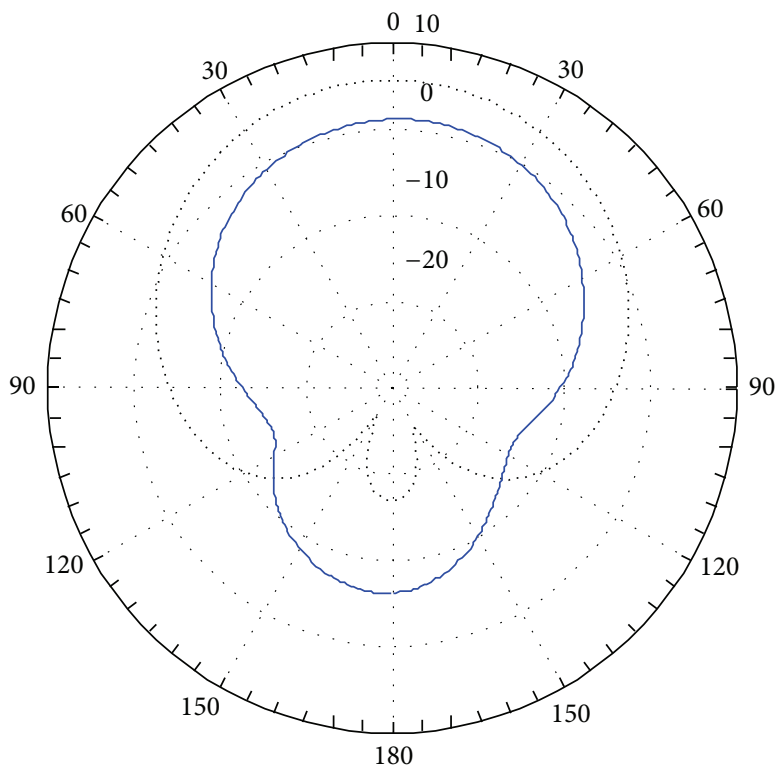

M. P. designed

M. P. measured

FIGURE 9: Simulation and measurement results for $H$-plane gain of miniaturized patch with longer feed line (frequency: $5.51 \mathrm{GHz}$ ).

Table 2 gives a summary of the performances of the traditional patch and miniature antennas. The miniaturization of the patch is achieved at the expense of acceptable degradation in gain, efficiency, and bandwidth. 


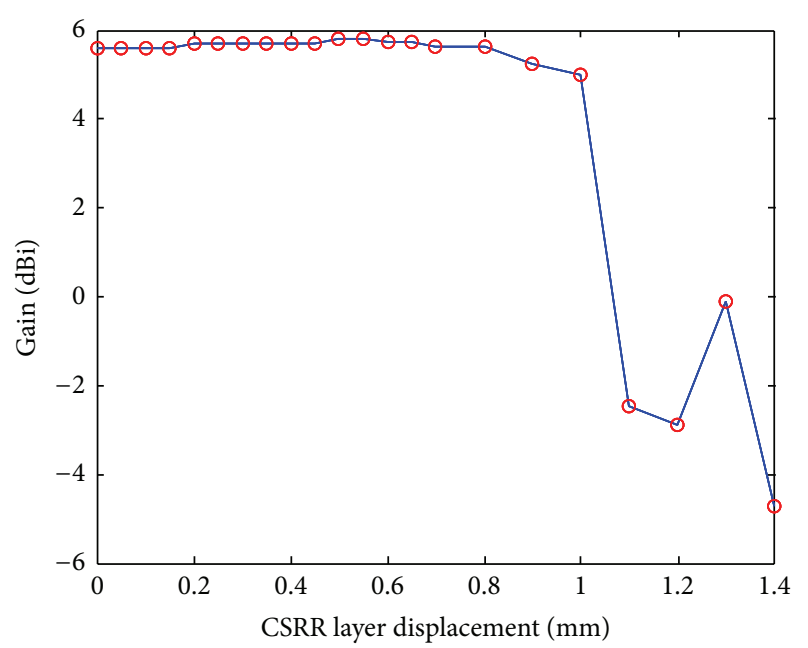

Figure 10: The simulation results showing the variation of the antenna gain due to the displacement (misalignment) of the CSRR layer along the feed line.

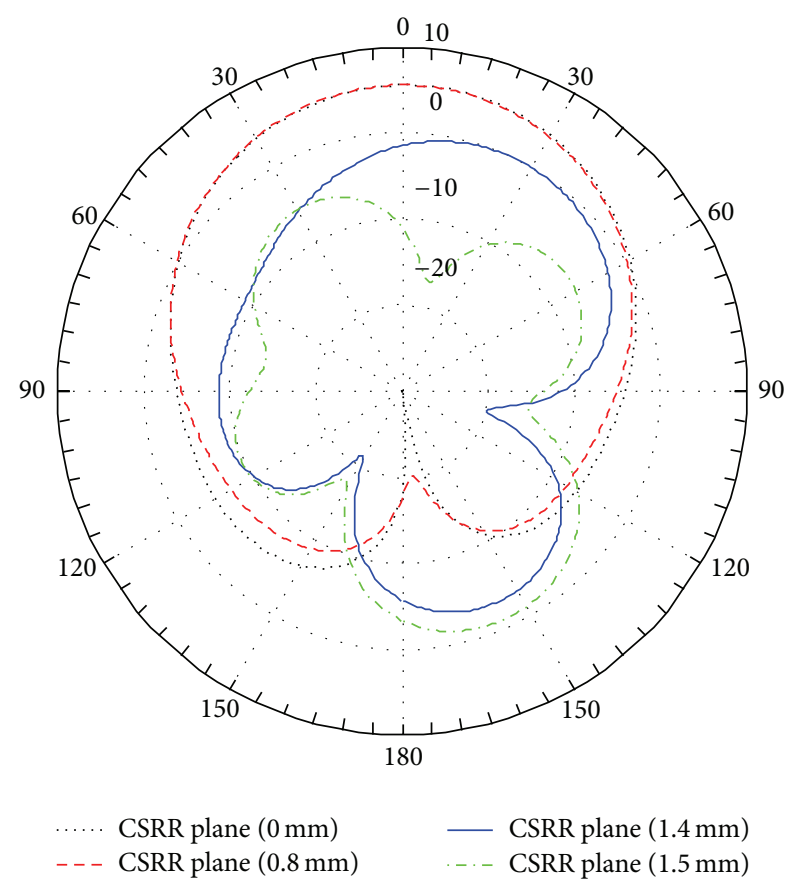

FIGURE 11: Simulation results for $E$-plane gain of miniaturized patch at different location of CSRR plane moved towards the feed line.

\section{Modeling of Miniaturized Patch Loaded with CSRR Layer}

In order to assess the operating principle and define a guideline to design a miniaturized antenna, first a transmission line loaded with CSRR plane shown in Figure 13 is simulated. The circuit model given in Figure 14 is employed to verify the operation of CSRR plane [22]. Figures 15 and 16 show the reflection and transmission coefficient results where a good agreement is noted between the simulations and model.

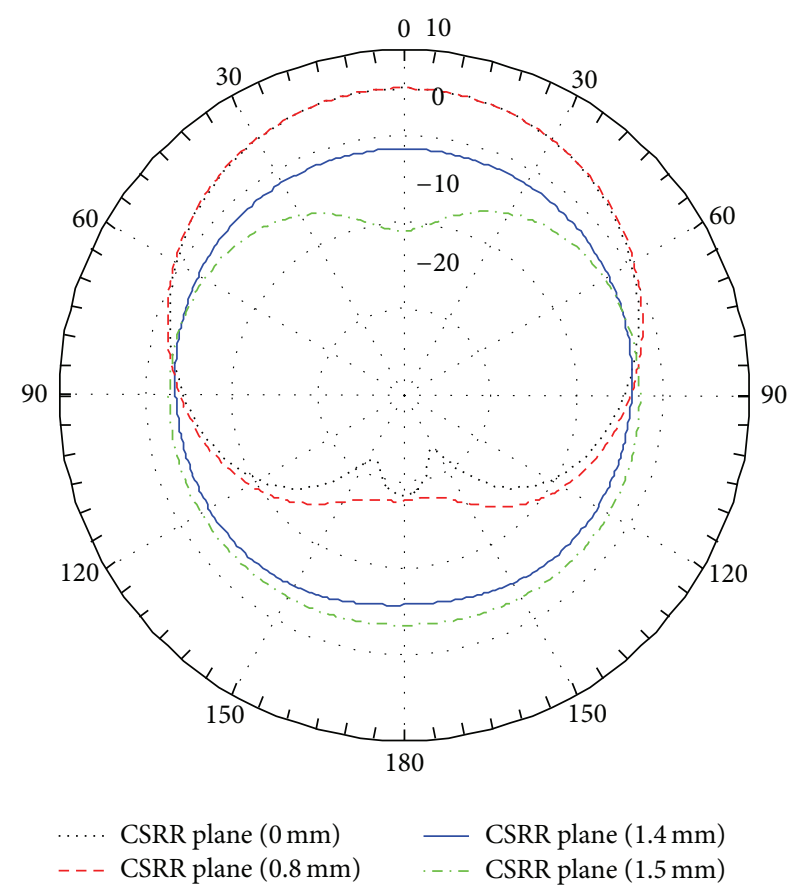

FIGURE 12: Simulation results for $H$-plane gain of miniaturized patch at different location of CSRR plane moved towards the feed line.

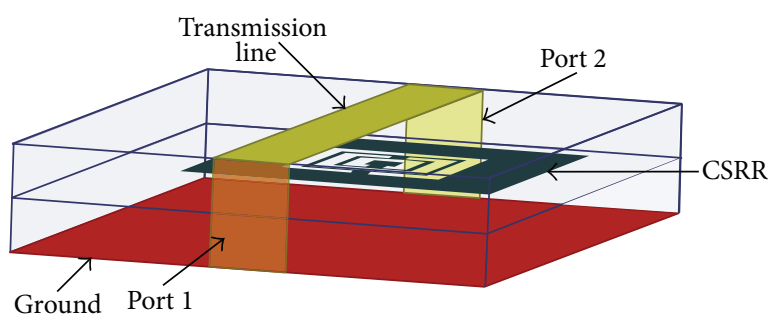

FIgURE 13: Transmission line loaded with CSRR.

TABLE 2: Comparison of performances of the traditional and miniaturized patch antennas obtained through simulations.

\begin{tabular}{lcc}
\hline & Traditional patch & Miniaturized patch \\
\hline Gain $(\mathrm{dBi})$ & 7.35 & 5.72 \\
Directivity (dBi) & 7.39 & 6.78 \\
Rad. efficiency (\%) & 98.6 & 78 \\
Bandwidth (\%) & 2 & 0.4 \\
Bandwidth (10 dB) $(\mathrm{MHz})$ & 103.4 & 21.2 \\
\hline
\end{tabular}

Prior to modeling of miniaturized square patch, the traditional patch is modeled using transmission line model of patch as shown in Figure 17 where the two radiating slots along the length of patch are modeled as shunt reactances. Figures 18 and 19 compare the magnitude and phase of reflection coefficient of circuit model and simulated results, respectively. The model results show a very good agreement with the simulated results.

Similar to the traditional patch antenna, the miniaturized one is also modeled with two radiating slots loaded with CSRR as shown in Figure 20. It is sufficient to model 


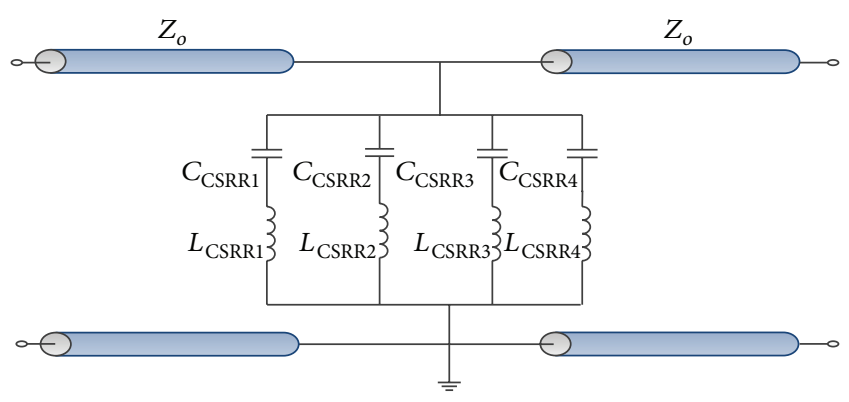

FIGURE 14: Circuit model of a transmission line loaded with CSRR.

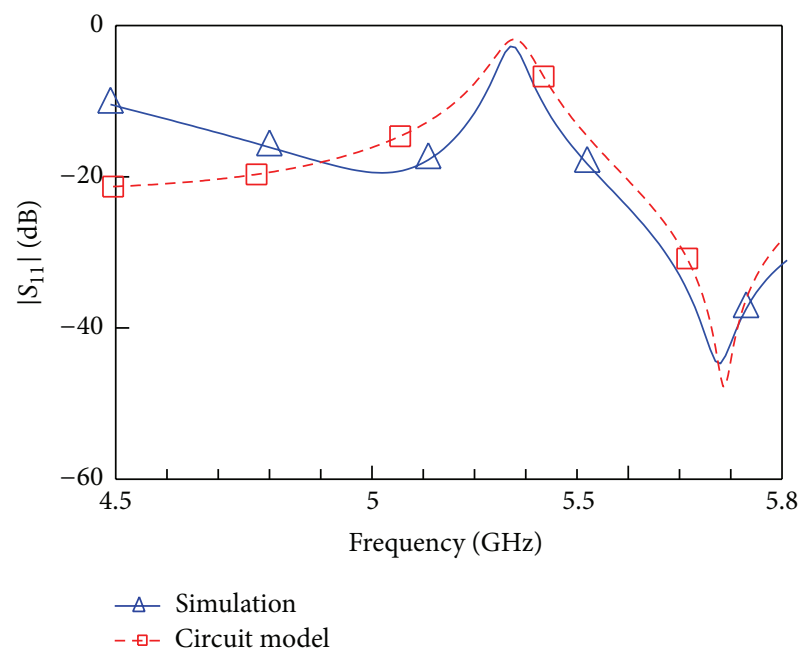

FIGURE 15: Comparison of circuit model and simulated reflection coefficient results of transmission line loaded with CSRR.

TABLE 3: Circuit modeling parameters of traditional patch and miniaturized patch antenna.

\begin{tabular}{lccc}
\hline $\begin{array}{l}\text { Miniaturized patch circuit } \\
\text { model parameters }\end{array}$ & \multicolumn{2}{l}{$\begin{array}{l}\text { Traditional patch circuit model } \\
\text { parameters }\end{array}$} \\
\hline$Z_{o}(\Omega)$ & 40 & $Z_{o}(\Omega)$ & 40 \\
$l_{o}(\mathrm{~mm})$ & 1 & $l_{o}(\mathrm{~mm})$ & 4 \\
$Z_{\text {otm }}(\Omega)$ & 90 & $Z_{o t}(\Omega)$ & 106 \\
$l_{\text {otm }}(\mathrm{mm})$ & 14.4 & $l_{o t}(\mathrm{~mm})$ & 9 \\
$Z_{o m}(\Omega)$ & 3.97 & $Z_{o p}(\Omega)$ & 17.5 \\
$l_{o m}(\mathrm{~mm})$ & 8 & $l_{o p}(\mathrm{~mm})$ & 15.95 \\
$R_{p m}(\Omega)$ & 500 & $R_{p}(\Omega)$ & 915 \\
$C_{p m}(\mathrm{pF})$ & 9.8 & $C_{p}(\mathrm{pF})$ & 0.44 \\
$C_{\mathrm{CSRR}}(\mathrm{pF})$ & 16.7 & - & - \\
$L_{\mathrm{CSRR}}(\mathrm{pH})$ & 57 & - & - \\
\hline
\end{tabular}

the CSRR in miniaturized patch with a single $L C$ section whose resonant frequency lies in the regime of resonant frequency of the antenna. Table 3 shows the summary of modeling parameters of traditional and miniaturized patch antennas. Figures 21 and 22 compare the magnitude and phase of reflection coefficient of model and simulated results, respectively. The miniaturized patch model results agree well

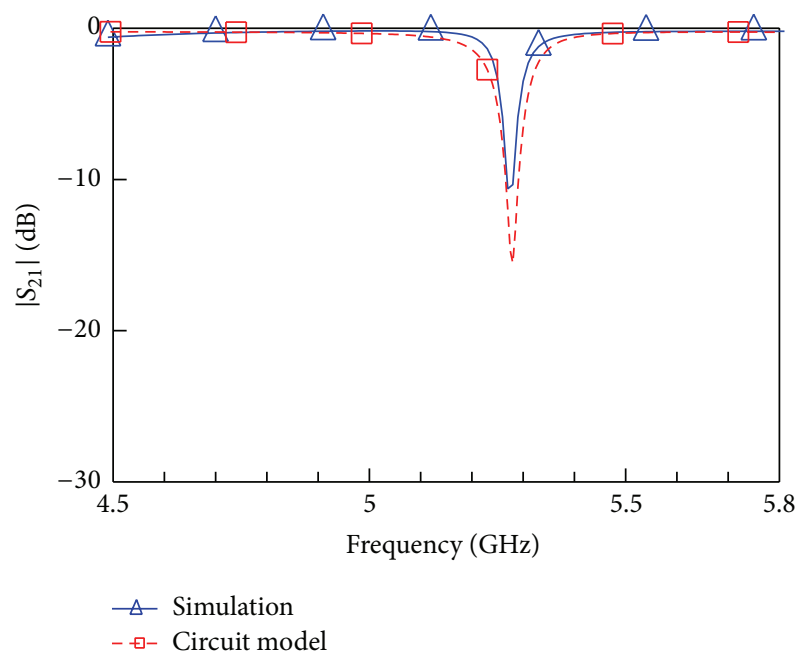

FIGURE 16: Comparison of circuit model and simulated transmission coefficient results of transmission line loaded with CSRR.

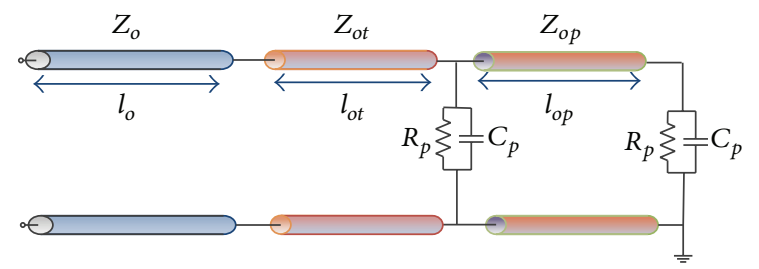

FIgURE 17: Circuit model of traditional patch antenna using transmission lines.

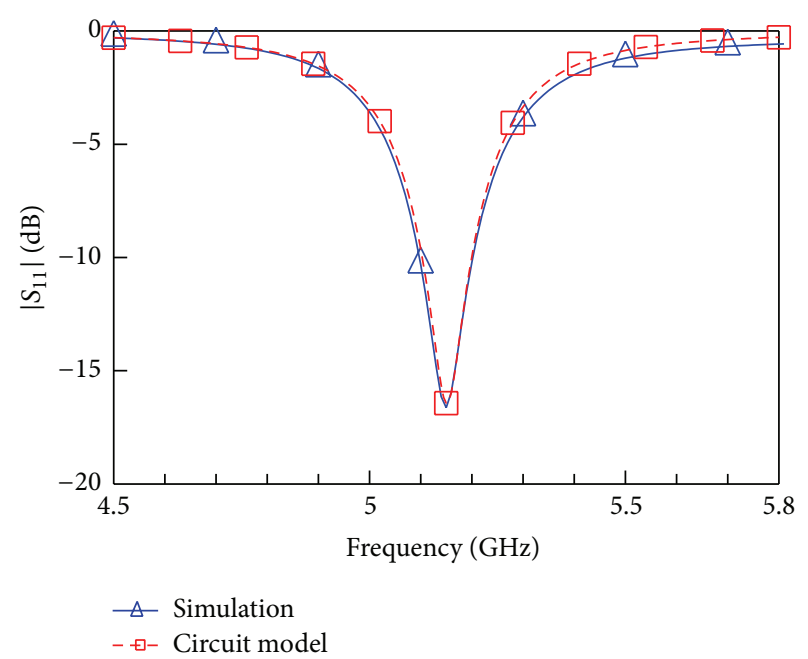

FIGURE 18: Comparison of circuit model and simulated reflection coefficient results of traditional patch antenna.

with the simulation results. Figure 23 shows the surface current, $J_{s}$, distribution on traditional square patch and miniaturized patch. The CSRR is changing the current distribution on the miniaturized patch which, in turn, changes the input impedance of the miniaturized patch. It also explains the reason behind the miniaturization of the square. The CSRR plane causes the current on the square patch to follow a longer 


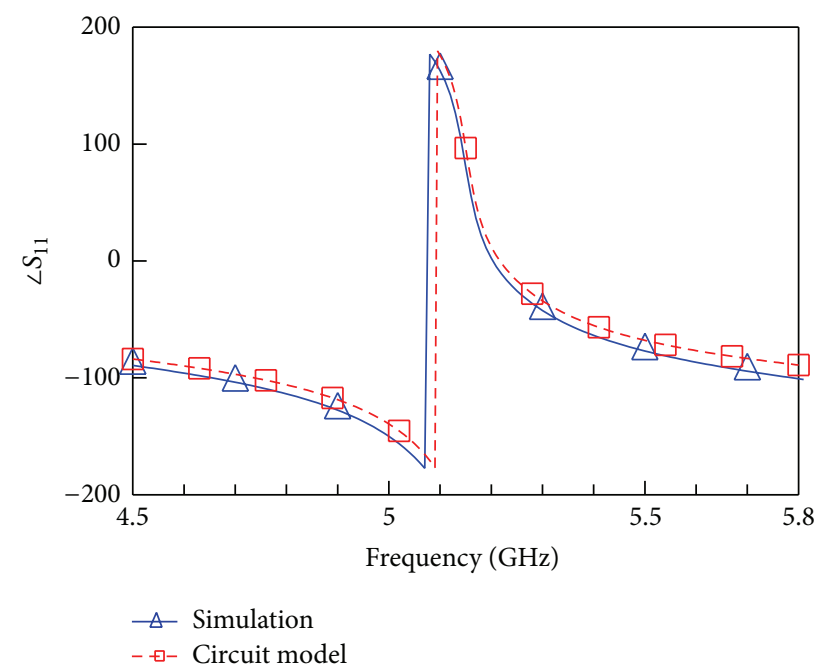

FIGURE 19: Comparison of circuit model and simulated phase of reflection coefficient results of traditional patch antenna.

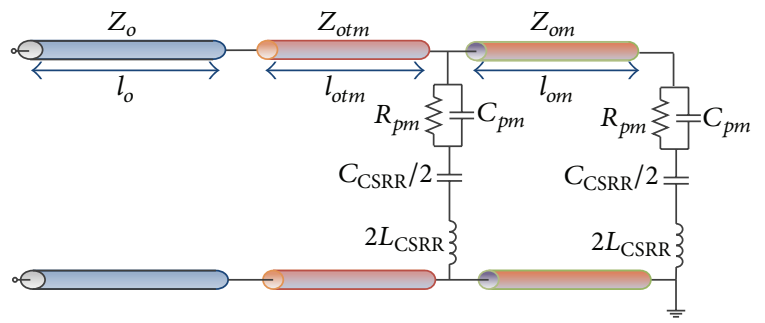

FIGURE 20: Circuit model of miniaturized patch loaded with CSRR using transmission lines.

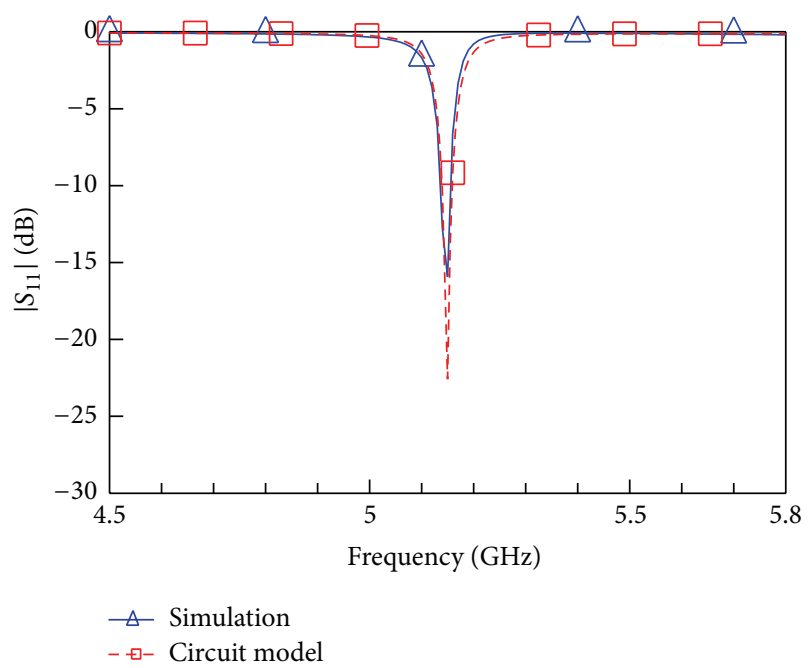

FIGURE 21: Comparison of circuit model and simulated reflection coefficient results of miniaturized patch antenna loaded with CSRR.

path allowing miniaturization. The current distribution also explains the degradation of the gain of the miniaturized antenna with the slight change of the CSRR plane location since the current distribution on the miniaturized antenna is highly dependent on the location of CSRR as shown in Figure 23. It should be noted that if the location of the CSRR

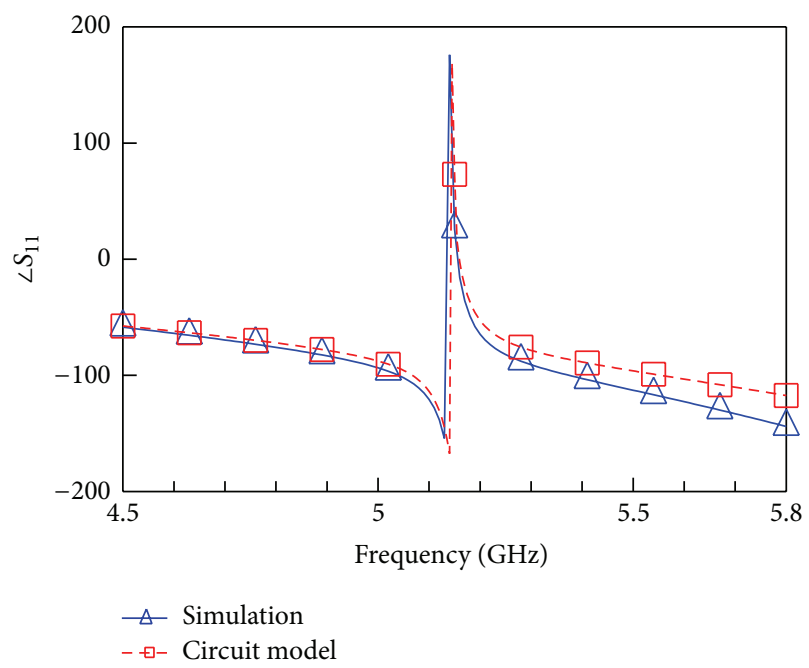

FIgURE 22: Comparison of circuit model and simulated phase of reflection coefficient results of miniaturized patch antenna loaded with CSRR.

is changed, it modifies the current distribution on antenna which eventually affects the far field radiation and gain of the antenna. Figure 24 shows current distribution on different locations of CSRR plane shifted towards the feed line of miniaturized antenna. The current distribution is highly dependent on the location of CSRR. As the CSRR plane is moved towards feed line, it starts loading the feed line. After $1 \mathrm{~mm}$ of shift, it is deduced that the maximum current distribution is being concentrated around CSRR towards the feed line which, in turn, affects the gain and radiated power of the antenna. Furthermore, as the current distribution on patch changes, the input impedance of patch starts to change. For a shifted CSRR plane, we need a slight modification in the matching network. However, our matching is fixed and designed for a CSRR positioned with reference to the center of the patch.

\section{Conclusion}

This paper presents simple yet effective method for the miniaturization and modeling of the patch antennas. Miniaturization of the square patch antenna is achieved by employing a horizontal layer of CSRR in between the patch and ground plane. The measurements results of the miniaturized antennas indicate that CSRR based loading plane shrinks the antenna area to $1 / 4$ of a traditional patch without sacrificing the antenna performances significantly. It is also demonstrated that the CSRR loaded antenna is very sensitive to the air gap in between the two substrates indicating that the antenna can be reconfigured using reactive loading elements on this plane.

\section{Conflict of Interests}

The authors declare that there is no conflict of interests regarding the publication of this paper. 

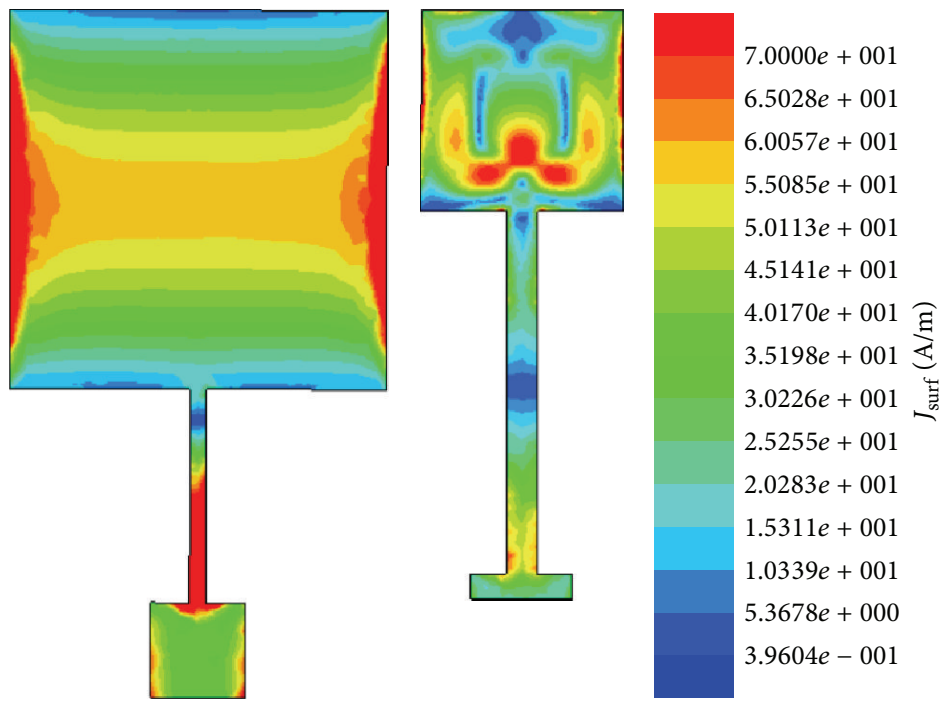

FIGURE 23: Comparison of surface current $\left(J_{s}\right)$ distribution of traditional patch and miniaturized patch loaded with CSRR.
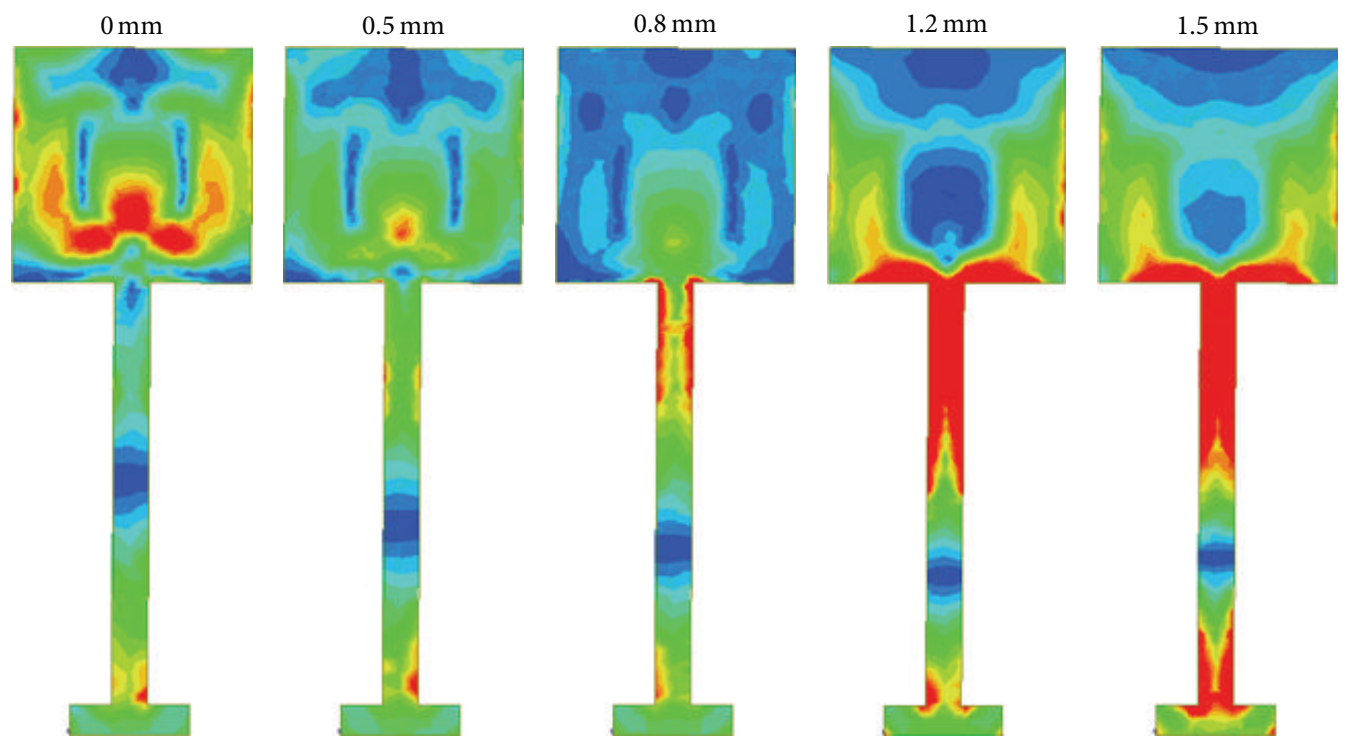

FIGURE 24: Current distribution on the miniaturized patch antenna on different location of CSRR layer.

\section{Acknowledgments}

The authors would like to thank Dr. Volkan Akan, Suleyman Kose, Ilhan Bayramoglu, and Dr. Evren Ekmekci for the fabrication, the measurement of the antennas, and fruitful discussions.

\section{References}

[1] D. M. Pozar and D. H. Schaubert, Microstrip Antennas, John Wiley \& Sons, 1995.

[2] R. Waterhouse, "Small microstrip patch antenna," Electronics Letters, vol. 31, no. 8, pp. 604-605, 1995.

[3] Y. Lee, S. Tse, Y. Hao, and C. G. Parini, "A compact microstrip antenna with improved bandwidth using complementary splitring resonator (CSRR) loading," in Proceedings of the IEEE
Antennas and Propagation Society International Symposium, pp. 5431-5434, IEEE, Honolulu, Hawaii, USA, June 2007.

[4] R. k. Baee, G. Dadashzadeh, and F. G. Kharakhili, "Using of CSRR and its equivalent circuit model in size reduction of microstrip antenna," in Proceedings of the Asia-Pacific Microwave Conference (APMC '07), pp. 1-4, IEEE, Bangkok, Thailand, December 2007.

[5] S. Lee, J. Woo, M. Ryu, and H. Shin, "Corrugated circular microstrip patch antennas for miniaturization," Electronics Letters, vol. 38 , no. 6, pp. 262-263, 2003.

[6] L. K. Wong and J. Y. Wu, "Single-feed small circularly polarised square microstrip antenna," Electronics Letters, vol. 33, no. 22, pp. 1833-1834, 1997.

[7] R. O. Ouedraogo, E. J. Rothwell, A. R. Diaz, K. Fuchi, and A. Temme, "Miniaturization of patch antennas using a 
metamaterial-Inspired technique," IEEE Transactions on Antennas and Propagation, vol. 60, no. 5, pp. 2175-2182, 2012.

[8] H. Mosallaei and K. Sarabandi, "Antenna miniaturization and bandwidth enhancement using a reactive Impedance substrate," IEEE Transactions on Antennas and Propagation, vol. 52, no. 9, pp. 2403-2414, 2004.

[9] Y. Dong, H. Toyao, and T. Itoh, "Design and characterization of miniaturized patch antennas loaded with complementary split-ring resonators," IEEE Transactions on Antennas and Propagation, vol. 60, no. 2, pp. 772-785, 2012.

[10] X. Zhao, Y. Lee, and J. Choi, "Design of a compact patch antenna using split-ring resonator embedded substrate," Microwave and Optical Technology Letters, vol. 53, no. 12, pp. 2786-2790, 2011.

[11] Y. Lee and Y. Hao, "Characterization of microstrip patch antennas on metamaterial substrates loaded with complementary split-ring resonators," Microwave and Optical Technology Letters, vol. 50, no. 8, pp. 2131-2135, 2008.

[12] S. Islam and M. Latrach, "Design construction and testing compact size patch antenna for RFID applications," Microwave and Optical Technology Letters, vol. 55, no. 12, pp. 2920-2925, 2013.

[13] A. Suntives and R. Abhari, "Miniaturization and isolation improvement of a multiple-patch antenna system using electromagnetic bandgap structures," Microwave and Optical Technology Letters, vol. 55, no. 7, pp. 1609-1612, 2013.

[14] Y.-J. Park, A. Herschlein, and W. Wiesbeck, "A photonic bandgap (PBG) structure for guiding and suppressing surface waves in millimeter-wave antennas," IEEE Transactions on Microwave Theory and Techniques, vol. 49, no. 10, pp. 1854-1859, 2001.

[15] J. Colburn and Y. Rahmat-Samii, "Patch antennas on externally perforated high dielectric constant substrates," IEEE Transactions on Antennas and Propagation, vol. 47, no. 12, pp. 1785-1794.

[16] J. Baena, J. Bonache, F. Martin et al., "Equivalent-circuit models for split-ring resonators and complementary splitring resonators coupled to planar transmission lines," IEEE Transactions on Microwave Theory and Techniques, vol. 53, no. 4, pp. 1451-1461, 2005.

[17] J. Bonache, M. Gil, I. Gil, J. Garcia-Garcia, and F. Martin, "On the electrical characteristics of complementary metamaterial resonators," IEEE Microwave and Wireless Components Letters, vol. 16, no. 10, pp. 543-545, 2006.

[18] M. S. Alam, N. Misran, B. Yatim, and M. T. Islam, "Development of electromagnetic band gap structures in the perspective of microstrip antenna design," International Journal of Antennas and Propagation, vol. 2013, Article ID 507158, 22 pages, 2013.

[19] http://www.lpkfusa.com/.

[20] S. Shi, W. Che, W. Yang, and Q. Xue, "Miniaturized patch antennas with enhanced bandwidth based on signal-inteference feed," IEEE Antennas and Wireless Propagation Letters, vol. 14, pp. 281-284, 2015.

[21] J. Tang, R. O. Ouedraogoand, A. R. Diaz, and K. Fuchi, "A continuously tunable miniaturized patch antenna," IEEE Transactions on Antennas and Propagation, vol. 13, pp. 10801083, 2014.

[22] A. Mehdipour, T. A. Denidni, and A. Sebak, "Multi-band miniaturized antenna loaded by ZOR and CSRR metamaterial structures with monopolar radiation pattern," IEEE Transactions on Antennas and Propagation, vol. 62, no. 2, pp. 555-562, 2014. 

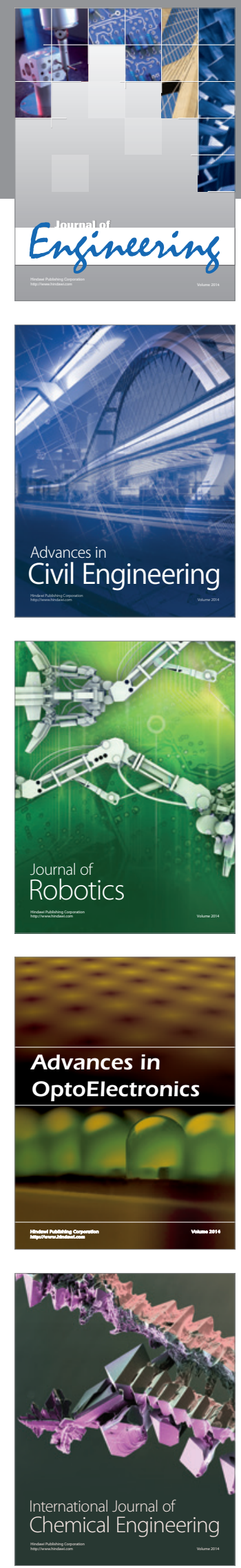

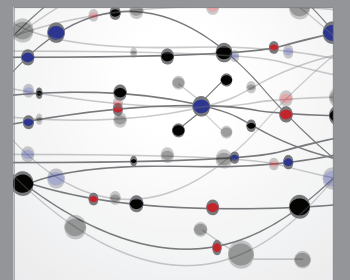

The Scientific World Journal
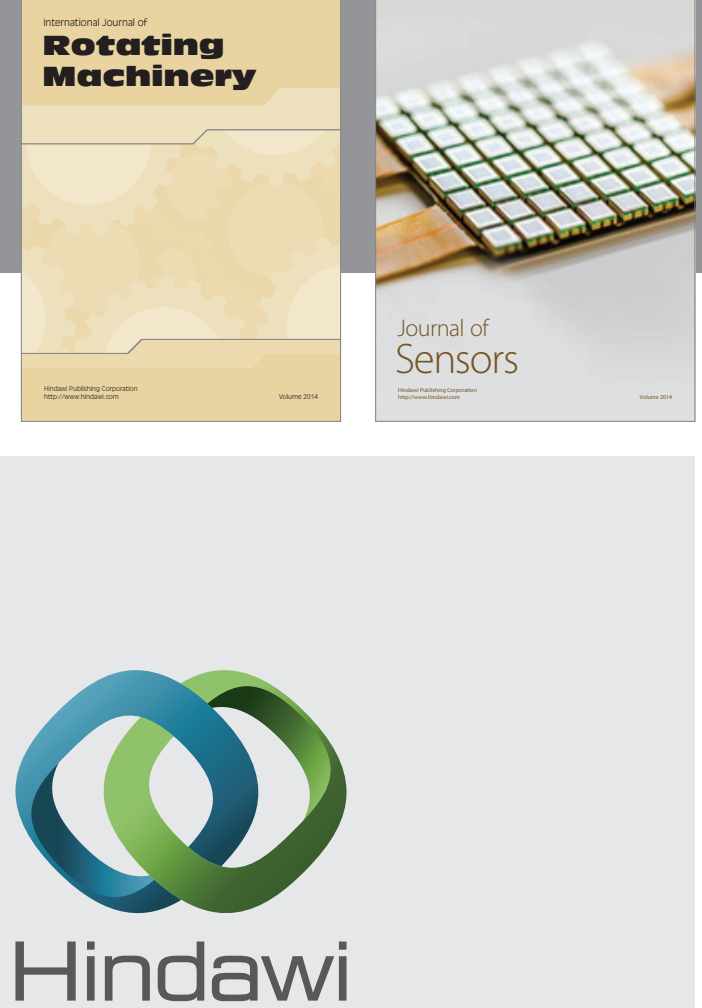

Submit your manuscripts at http://www.hindawi.com
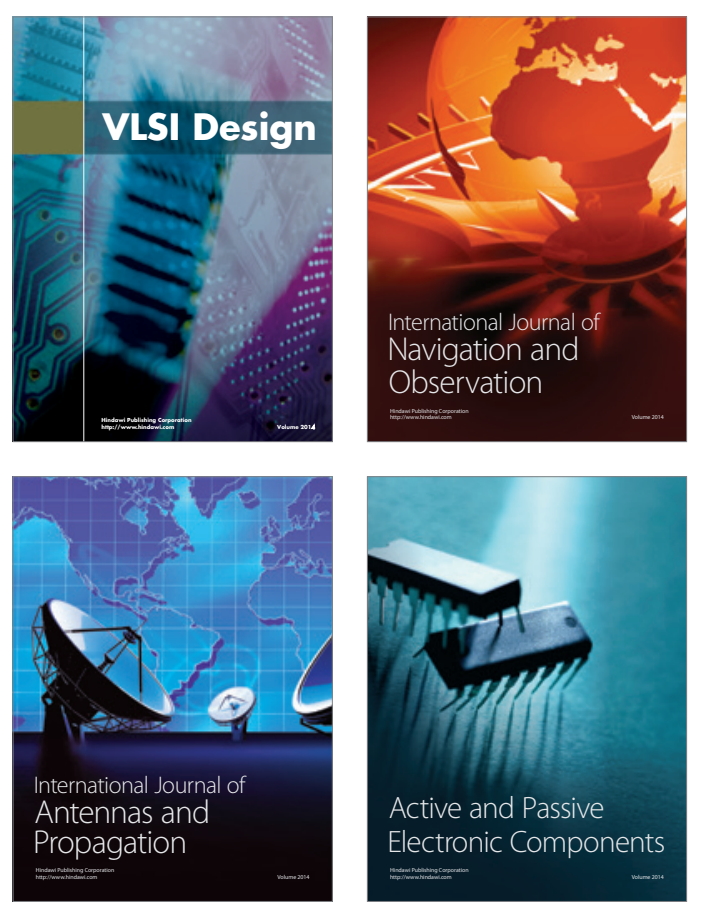
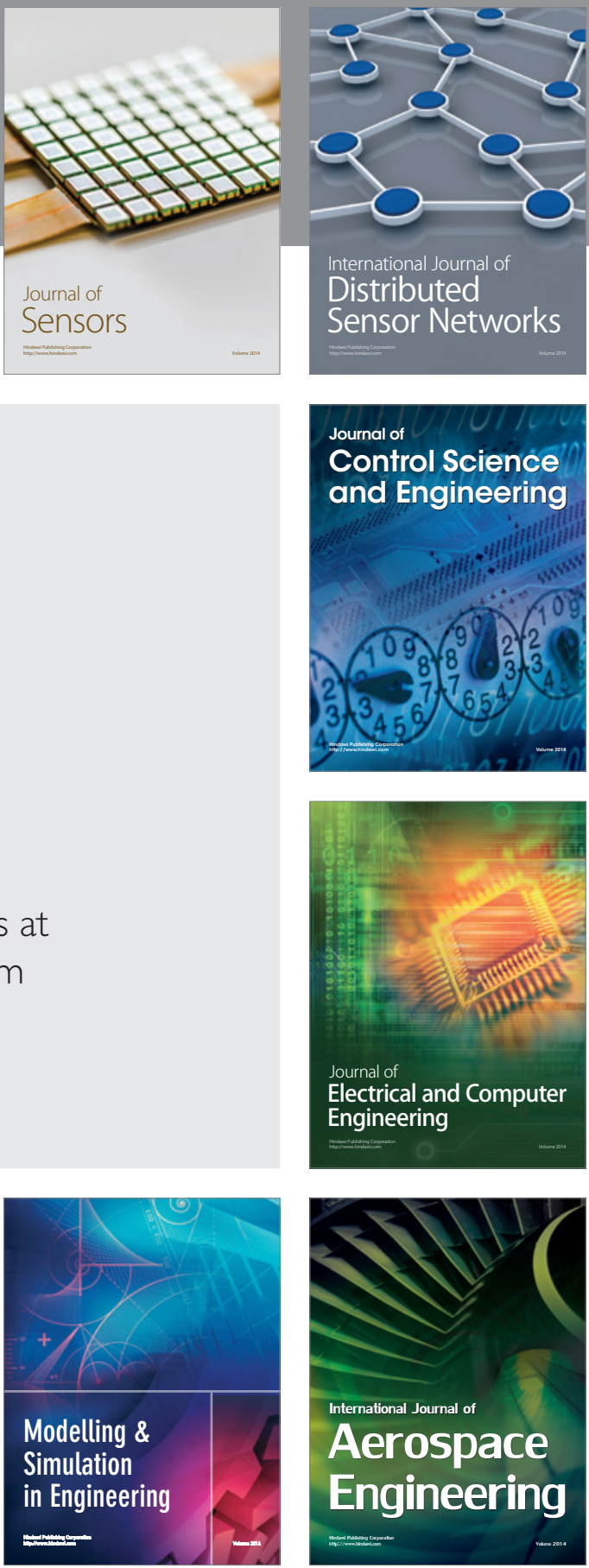

Journal of

Control Science

and Engineering
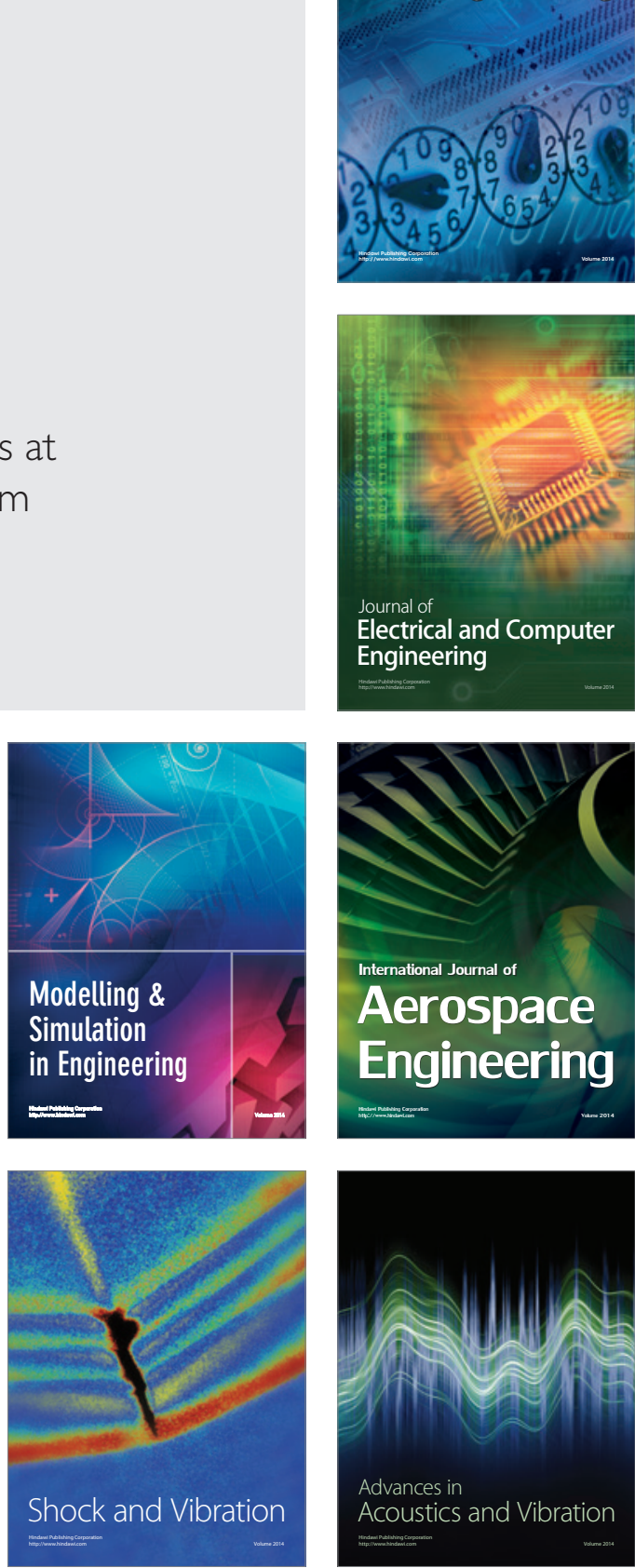